\title{
Temporal and structural characteristics of a two dimensional gas of hard needles
}

\author{
M. Ebrahim Foulaadvand ${ }^{1,2}$ and Mohsen Yarifard ${ }^{1}$ \\ 1 Department of Physics, Zanjan University, P.O. Box 19839-313, Zanjan, Iran. and \\ 2 Computational Physical Sciences Laboratory, Department of Nano-Science, \\ Institute for Research in Fundamental Sciences (IPM), P.O. Box 19395-5531, Tehran, Iran.
}

(Dated: November 7, 2018)

\begin{abstract}
We have simulated the dynamics of a $2 \mathrm{D}$ gas of hard needles by event-oriented molecular dynamics. Various quantities namely translational and rotational diffusion constants and intermediate self scattering function have been explored and their dependence on density is obtained. Despite absence of positional ordering, the rotational degree of freedom behaves nontrivially. Slowing down is observed in the angular part of the motion. It is shown that above a certain density the rotational mean squared displacement exhibits a three stage regime including a plateau.
\end{abstract}

\section{INTRODUCTION}

Molecular dynamics (MD) simulation of anisotropic hard objects such as ellipsoids and spherocylinders has been the subject of exploration in past decades [1]. It has been shown the alignment of non-spherical molecules can lead to a diversity of phases, mainly orientational in nature, in liquid crystals [1-3]. Despite the profound insight obtained via tremendous Monte Carlo (MC) simulations of the static phases, many dynamical, transportational and structural properties namely kinetic arrest and glassy behaviours have only been poorly understood [4]. In spite of employment of other simulation techniques like Brownian dynamics $[5-7 \mid$ and theoretical approaches such as kinetic theory [8, 9], density functional [10-12] and hydrodynamics equations approach [13], event-oriented MD remains as an efficient tool for probing the dynamical aspects of hard gases of nonspherical objects. Among elongated and anisotropic hard bodies, infinitely thin needles have received quite notable attention [1]. The first MD simulation attempt were carried out for a three dimensional gas of hard needles by Frenkel and Maguire [14, 15] and Magda et al. [16]. In these investigations, various temporal auto-correlations were explored and compared to predictions of Enskog and Doi-Edward theories. The physics of hard needle gas is sensitive to dimensionality. By extensive MC simulations in 2D, Frenkel and Eppenga showed the existence of quasi long range order in the system at high densities [17]. It was argued that this $2 \mathrm{D}$ system undergoes a Kosterlitz-Thouless phase transition 18]. Resurgence of interest in the dynamical properties of hard needle gas was sparked by papers of Renner et al. [19] and Obukhov et al. [20] who introduced a rotator model. Their simple ideal glass former can mimic the basic dynamical properties of an orientational glass. This rotator model was shown to exhibit orientational glassy behaviour like its positional counterpart i.e., hard-sphere system [21]. A natural question to ask is whether the orientational glassy behaviour survives if one releases the positional degrees of freedom. Chrzanowska et al. carried out the first MD simulation of a two dimensional hard needle gas [22, 23] and mainly studied velocity auto correlations whereas the transport properties remained unexplored. Subsequently transport coefficients like self-diffusion and shear viscosity of a 3D hard needle gas were studied within MD approach by Mukôyama et al. [24, 25]. We wish to note that the hard needle rotator model is amenable to comparison with experiments carried out via neutron scattering and has shown to successfully model the experimentally observed phenomenon of orientation glassy dynamics [26]. Recent investigations on 2D hard needle gas involve deposition-evaporation dynamics [27] and restriction of needles center of masses [28]. Here we focus on the transportation features to illuminate the interplay of translational and rotational degrees of freedom in 2D systems of highly anisotropic hard objects.

\section{DESCRIPTION OF THE PROBLEM}

Consider a system comprising of infinitely thin hard needles with mass $m$ and length $l$ which are restricted to move in the two dimensional $x-y$ plane. The interaction between needles is assumed to be hard core. We work in the reduced units in which $m$ and $l$ are taken unity. Upon a collision between two needles, the centre of mass $(\mathrm{CM})$ velocity and the angular velocity around the $z$ axis through the $\mathrm{CM}$ will change. We assume the collisions are elastic and frictionless therefore the energy (entirely kinetic) is conserved after a collision. The kinetics of such collision has been investigated in detail in [23]. We study the dynamics of this $2 \mathrm{D}$ needle gas within the collision-oriented MD approach. In the collision-oriented MD the evolution of system takes place from collision to collision. The main task in MD of hard objects is to find the collision time between two apart objects [29]. A numerical scheme has been originally introduced in [30, 31]. The method is based on finding a geometrical condition for overlapping between objects. This condition is given as $H_{1}, H_{2}<\frac{l}{2}|\sin (\theta)|$ where $\theta$ is the angle between needles. $H_{2}$ is the distance of needle $2 \mathrm{CM}$ to the line of needle 1 and reverse. To find the next collision time we move all the needles in small time steps until the needles become very close to each other that can be regarded as touching. Then we update the velocity and angular velocity of the colliding needles and proceed to the next 


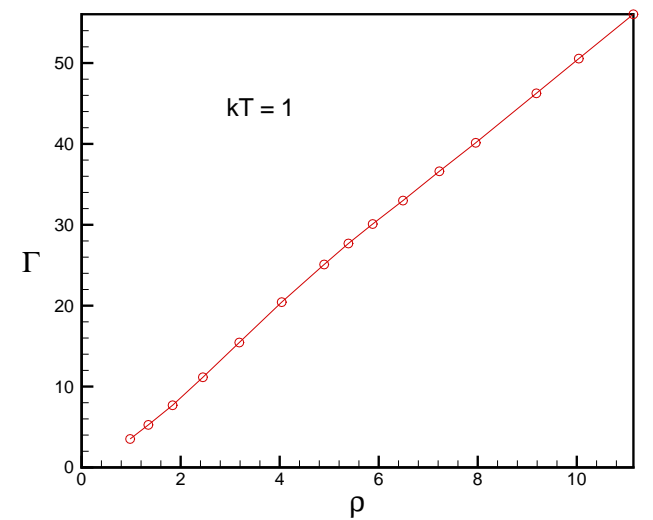

FIG. 1: Fig.1: Collision frequency $\Gamma$ vs $\rho$. The dependence is linear like $3 \mathrm{D}$ gas of hard needles investigated in [15, 16]. The slope changes around $\rho=5.5$. Line is for helping eyes

collision event.

\section{MOLECULAR DYNAMICS SIMULATION}

\section{A. Static properties and velocity autocorrelations}

We have simulated the dynamics of a $2 \mathrm{D}$ gas of hard needles with the method explained in section II. Periodic boundary condition is imposed. The size of our simulation box is set to $L=7$. The number density is defined as $\rho=\frac{N}{L^{2}}$ where $N$ is the number of needles. The total energy of the system $E$ (entirely kinetic) is divided into two segments of translational $E_{t r s}$ and rotational $E_{r o t}$. We remark that due to lack of any energy scale in the potential energy between particles, the temperature $T$ appears as an overall multiplicative factor in thermodynamic quantities such as pressure and free energy. Thus the state of the system trivially depends on temperature and hence energy. Nevertheless $E$ determines the time scale $\tau$. In the thermal unit one has $\tau=l \sqrt{\frac{m}{k_{B} T}}$. We have taken the energy per particle $\frac{E}{N}=1.5$ in our simulations which gives $k_{B} T=1$. A useful quantity is the average number of collision per unit time $\Gamma$ that a needle experiences. In [15] and [16] it is argued that $\Gamma$ has a linear dependence on number density $\rho$ in a $3 \mathrm{D}$ gas. According to Frenkel and Maguire arguments in 3D, $\Gamma$ scales as follows: $\Gamma \sim l^{2}\left(\frac{k T}{m}\right)^{\frac{1}{2}} \rho$. In fact the Doi-Edward theory predicts such linear behaviour as well. We have computed the dependence of $\Gamma$ on $\rho$ in our 2D model. Figure (1) depicts that $\Gamma$ has, analogous to the $3 \mathrm{D}$ case studied in [15, 16], a linear dependence on $\rho$. Note the slope change about $\rho=5.5$.

The equation of state i.e., dependence of $P$ on the $\rho$ is shown in figure (2). In fact $P$ does not linearly increases with $\rho$. The slope changes about the same value $\rho=$ 5.5 which is in agreement with MC results of Frenkel

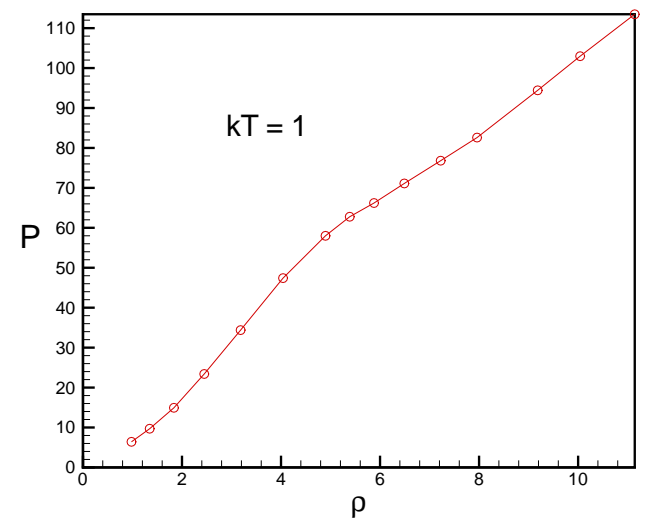

FIG. 2: Fig.2: System pressure $P$ (reduced units) vs $\rho$. Pressure slope changes around $\rho=5.5$. Line is for helping eyes.

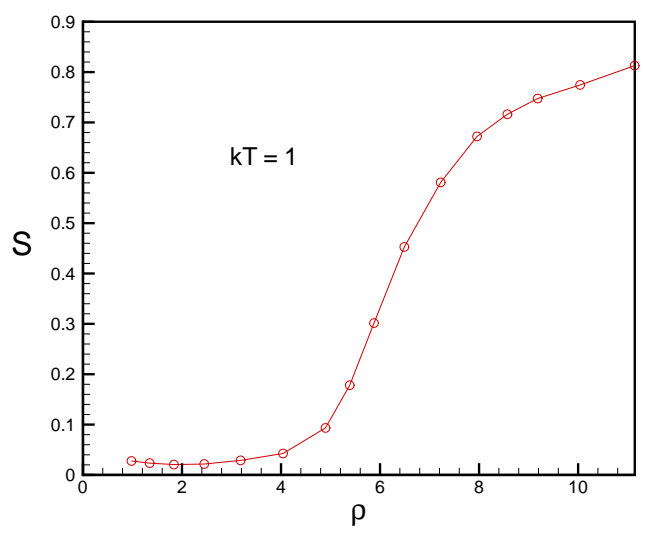

FIG. 3: Fig.3: Dependence of nematic order parameter $S$ on $\rho$. The transition to nematic ordered phase is expected to be a finite size effect. Line is for helping eyes.

and Eppenga [17]. This marks that from translational viewpoint the system is totally distinct from ideal gas.

Next we turn to order parameter. The orientational nematic order parameter $S$ is defined as follows:

$$
S=\frac{1}{N^{2}}\left\langle\sum_{i, j=1}^{N} \cos \left(2 \theta_{i}-2 \theta_{j}\right)\right\rangle .
$$

In which $\theta_{i}$ is the director angle of $i$-th needle with positive $x$ axis and the average is taken over trajectories. Dependence of $S$ on $\rho$ is sketched in figure (3).

Our simulation shows a large value for the nematic order parameter $S$ at high densities which corresponds to a nematic phase. This result is expected to be a finite-size effect. We argue that the possibility of truly phase transition to a nematic phase is not excluded in 2D. More concisely, the conditions of the Mermin-Wagner theorem are not satisfied here due to the fact that inter-molecular po- 
tential between needles $V(r, \theta)$ is not separable. Despite Monte Carlo simulations have not shown the existence of such isotropic-nematic transition in a $2 \mathrm{D}$ gas of needles 17], a quasi long range order of Kosterlitz-Thouless type [17, 18] was shown to persist in the system. The subject of temporal velocity auto correlation function has been the extensively studied in 15, 16] within the framework of MD and recently in [22, 23] both in event-oriented MD approach and analytically in the framework of Enskog kinetic theory. We have explored the autocorrelation between longitudinal and transverse decomposition of velocity. These quantities are defined as follows:

$$
\begin{gathered}
C_{\|}(t)=\frac{1}{\left\langle v^{2}(0)\right\rangle}\langle\vec{v}(t) \cdot \hat{u}(0) \vec{v}(0) \cdot \hat{u}(0)\rangle . \\
C_{\perp}(t)=\frac{1}{\left\langle v^{2}(0)\right\rangle}\langle\vec{v}(t) P \vec{v}(0)\rangle .
\end{gathered}
$$

$\hat{u}$ denotes the unit vector along the needle orientation and the matrix $P=1-\hat{u}(0) \hat{u}^{t}(0)$ is the projection operator. In figure (4) we exhibit the temporal dependence of $C_{\|}(t)$ and $C_{\perp}(t)$ for various densities.

We refer the readers to 22] for a detailed discussion on velocity autocorrelations. We now consider the temporal auto correlation of the second order angular order parameter $C_{2}(t)$. This quantity is defined as follows:

$$
C_{2}(t)=\left\langle P_{2}(\hat{u}(t) \cdot \hat{u}(0))\right\rangle .
$$

In which $P_{2}$ is the second order Legendre polynomial. Figure (5) shows the temporal dependence of $C_{2}(t)$.

In low densities we see a fast decay which is attributed to the fluid like behaviour. By increasing the density the temporal behaviour becomes slow and two characteristics time scale emerge. This confirms the nontrivial role played by the angular degree of freedom. We emphasize that simulation on larger system is needed to ensure the persistence of such large correlations. The intermediate self scattering function $F_{s}(q, t)$ is shown in figure (6).

\section{B. Structural properties}

We have obtained and explored various structural quantities. The radial distribution function $g(r)$ (not shown) is featureless for $r>1$ and regardless of the density value it approaches to unity without showing any significant amplitude fluctuations. This demonstrates that the gas posses no positional ordering. However, the existence of excluded volume effect discriminates its positional features to ideal gas. Next we consider the angular spatial correlation function $g_{2}(r)$. This quantity is defined as [17]: $g_{2}(r)=\langle\cos (2[\theta(r)-\theta(0)])\rangle$. The average is over all needle pairs having CM to CM distance $r$. Figure (7) plots the dependence of $g_{2}(r)$ vs $r$.
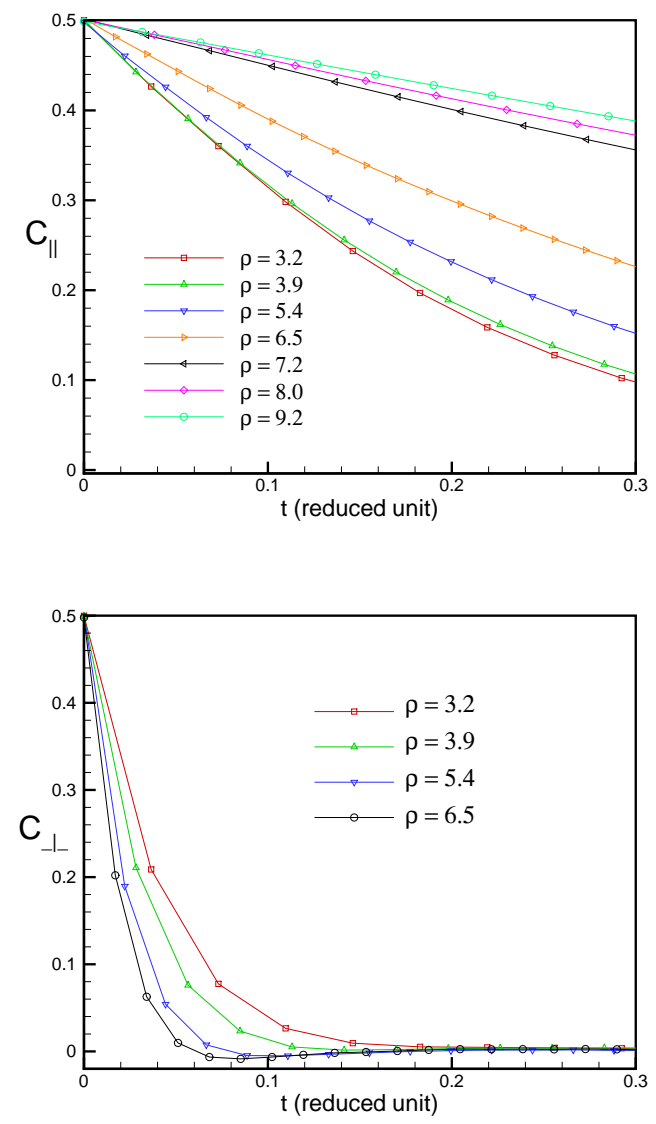

FIG. 4: Fig.4: Temporal dependence of longitudinal and transverse components of velocity ACF. Longitudinal component exhibits a slower decay which is due to direction of impulsive force between needles.

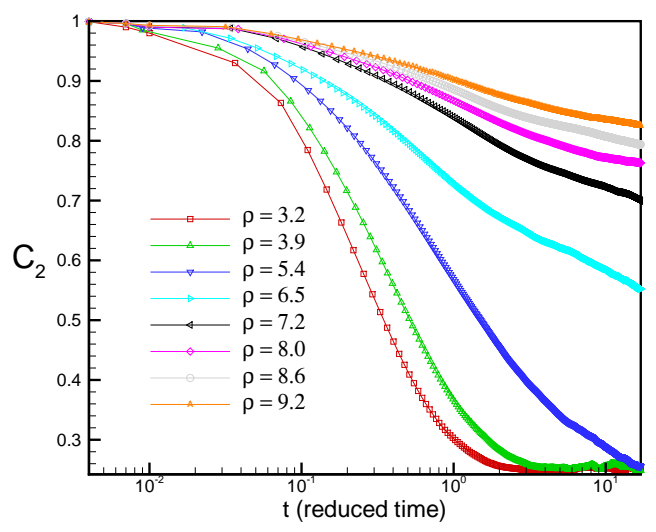

FIG. 5: Fig.5: Temporal dependence of $C_{2}$ at various densities (semi-log scale). 


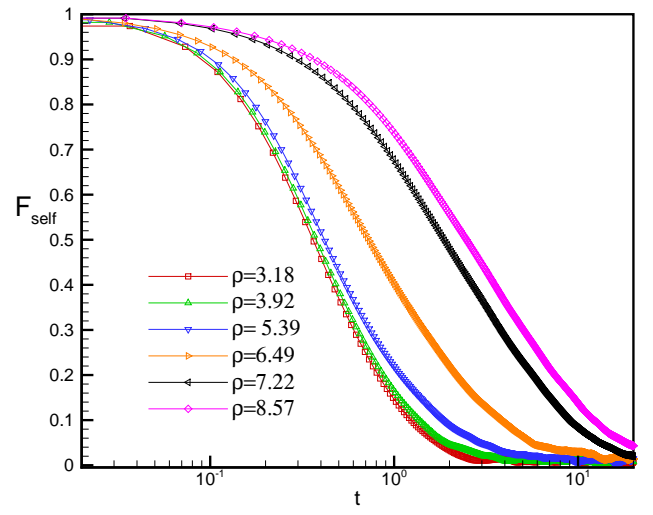

FIG. 6: Fig.6: Time dependence of the intermediate self scattering function $F_{s}(q, t)$ at $q=3$.

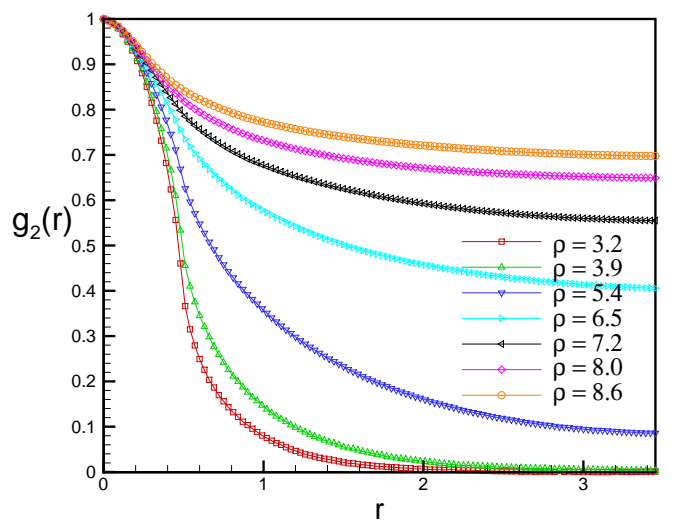

FIG. 7: Fig.7: Dependence of $g_{2}(r)$ on $r$ for various densities.

For small densities, $g_{2}(r)$ rapidly approaches zero. This decrease is faster than algebraic. Contrary, for high density $\rho>6$ we have a slow decrease which can be indicative of slow dynamics and angular structural arrest. Taking the decay form algebraic as $g_{2}(r) \sim r^{-\eta_{2}}$ we have found out by fitting a curve the decay exponents at various densities. Figure (8) shows our exponents which are compared to those of Frenkel and Eppenga [17]. At high densities $\mathrm{MD}$ and $\mathrm{MC}$ results are in good agreement while quite notable differences are seen at low densities.

\section{TRANSPORT PROPERTIES}

Now we report our results for the transport properties and orientational structure of the system. We begin with translational diffusion coefficient $D_{\text {trs }}$ defined as follows:

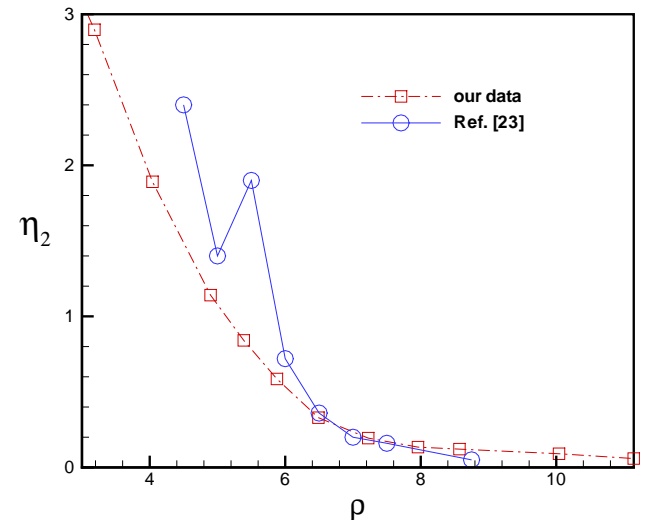

FIG. 8: Fig.8: Dependence of algebraic decay exponent $\eta_{2}$ on $\rho$.

$$
D_{\text {trs }}=\frac{1}{2 d N t}\left\langle\sum_{i=1}^{N}\left|\vec{r}_{i}(t)-\vec{r}_{i}(0)\right|^{2}\right\rangle
$$

The term in the bracket is the translational meansquare displacement (MSD) $\left\langle(\Delta \vec{r})^{2}\right\rangle$. Here the spatial dimension $d$ is two and the average is doubly taken over trajectories of needles' CM and time origins. Figure (9) sketches the time dependence of the translational MSD (see journal printed version to see figure nine). After a ballistic regime, one recovers normal diffusive behaviour. This confirms that from translational viewpoint, the system resembles an structureless gas. Figure (10) plots a CM trajectory of a typical needle at two densities $\rho=3.9$ and $\rho=7.9$ :

Three distinctive kinds of motion can be identified: diffusion, channeling and entanglement among cages formed by neighbouring needles. At low densities the motion resembles a normal diffusion. At higher densities a channeling type of motion emerges and at higher densities the topological constraints in 2D leads to entanglement. Let us now explore the rotational diffusion $D_{\text {rot }}$. In sharp contrast, $D_{\text {rot }}$ seems to be entirely of different nature. We have:

$$
D_{\text {rot }}=\frac{1}{2 \zeta N t}\left\langle\sum_{i=1}^{N}\left|\theta_{i}(t)-\theta_{i}(0)\right|^{2}\right\rangle
$$

Analogously the bracketed term denotes the angular mean square displacement $\left\langle(\Delta \theta)^{2}\right\rangle$ and $\zeta$ is the number of angular degrees of freedom (here $\zeta=1$ ). Figure (11) sketches the time dependence of $\left\langle(\Delta \theta)^{2}\right\rangle$ (see journal printed version to see figure 11). A significant difference is seen compared to the translational diffusion. For $\rho \geq 8$ the rotational MSD exhibits a three-stage regime which can be attributed to angular glassy dynamics. This is 

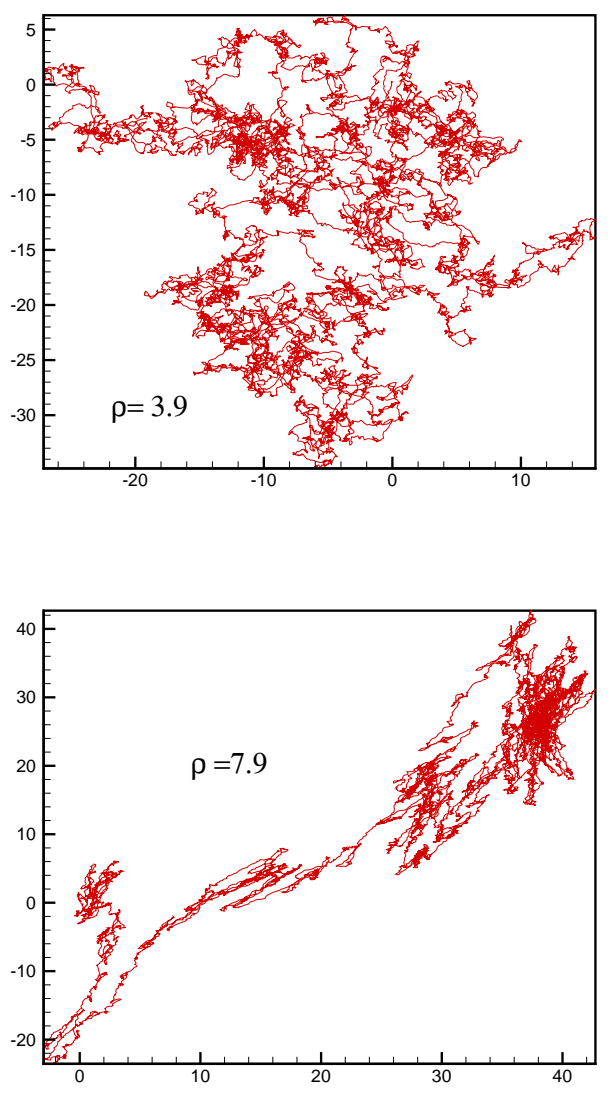

FIG. 9: Fig.10: CM trajectory of a needle at $\rho=3.9$ during 1586 time unit and $\rho=7.9$ during 2329 time unit. Note the unequal axes scaling. The true trajectories are elongated along the axis having larger scale.

consistent to the density proposed in [17]. The possibility of angular glassy behaviour has been earlier explored by Renner et al. [19]. It was shown above a $\rho_{c}$, the angular auto correlations exhibit slow dynamics and multi step relaxation which can be attributed to glassy behaviour. Our findings is supportive of the existence of angular glassy behaviour even when the translational degree of freedom is released. We stress that simulations with a larger system size is crucially needed to confirm this conclusion. Finally we discuss the dependence of translational and rotational diffusion constants on $\rho$ (figures 12 and 13).

For $\rho<5$ translational diffusion constant $D_{\text {trs }}$ shows a decreasing behaviour. This is in accordance with the Enskog kinetic theory [15]. In fact at low densities successive binary collisions are uncorrelated therefore both $D_{t r s}$ and $D_{\text {rot }}$ would be inversely proportional to $\rho[1]$. For large densities $D_{t r s}$ shows an increasing trend which is in qualitative agreement with the 3D results [16]. Frenkel and Maguire have exploited the Doi-Edwards theory, valid above semi-dilute concentrations, and developed a theory for the translational motion in 3D [15]. According

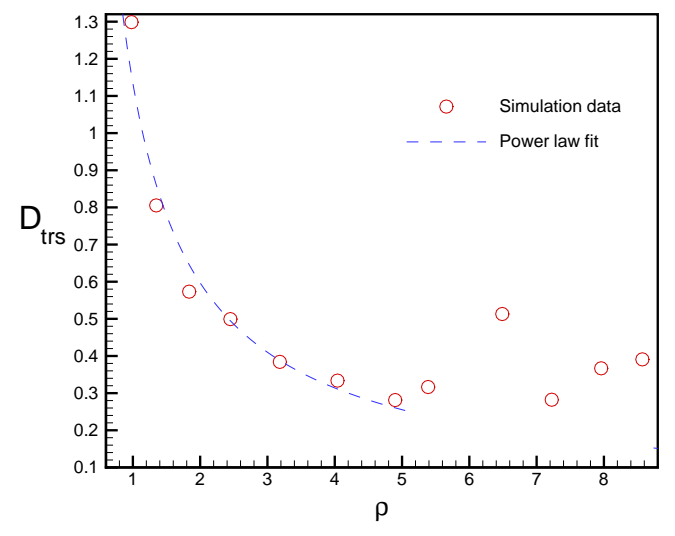

FIG. 10: Fig.12: Dependence of $D_{t r s}$ on needles density $\rho$.

to their results $C_{\|}$scales as $D_{\text {rot }}^{-0.5}$. Combining this with the scaling result $D_{\text {rot }} \sim \rho^{-2}$ and that $D_{\|}$is the integral of $C_{\|}(\tau)$ over time they concluded that parallel component of translational diffusion tensor scales as $D_{\|} \sim \rho^{\frac{1}{2}}$. Moreover, transverse component of diffusion tensor scales as $D_{\perp} \sim \rho^{-\frac{1}{2}}$ within Doi-Edward theory. Therefore they concluded that at high densities $D \sim \rho^{\frac{1}{2}}$. The MD data of Magda et al. 16] gives the dependence of $D_{\perp}$ on $\rho$ as $D_{\perp} \sim \rho^{-1.57}$. Our results in figure (12) is in qualitative agreement with the result obtained for a $3 \mathrm{D}$ gas of hard needles [15, 16, 24]. Note that $D=\frac{1}{2}\left(D_{\perp}+D_{\|}\right)$ and at high densities it is dominated by the longitudinal component. The fitted exponent to the portion $\rho \leq 5$ of our result for translational diffusion gives $D_{\text {trs }} \sim \rho^{-0.93}$. The value 0.93 slightly differs to the predicted exponent 1 by Enskog theory. This shows dimensionality notably affects the system properties. Eventually figure (13) exhibits the dependence of $D_{\text {rot }}$ on $\rho$. In 3D based on scaling arguments it can be concluded that within the Doi-Edward theory $D_{\text {rot }}$ scales as $\rho^{-2}$. MD simulations for a 3D gas of hard needles gives the dependence of $D_{\text {rot }}$ on density as $D_{\text {rot }} \sim \rho^{-\beta}$ with $\beta \in[1.8,2.2]$ [15]. The MD results of Magda et al. [16] give the exponent $\beta=1.5$ for $32 \leq \rho L^{3} \leq 72$ and $\beta=1.89$ for the density range $72 \leq \rho L^{3} \leq 100$ ( $L$ is the needle length). We have fitted both an algebraic curve $D_{\text {rot }} \sim \rho^{-\beta}$ and an exponential curve $D_{\text {rot }} \sim e^{-\frac{\rho}{\xi}}$ to our own data. It turned out that $\beta=3.6$ and $\xi=0.92$ with $\chi^{2}=0.7828$ and 0.9375 for the power law and exponential fits correspondingly (exponential curve is better fitted). We thus observe a slower decrease for $D_{\text {rot }}$ in 2D rather than in three. This can be explained on the basis of having more pronounced degree of entanglement and topological constraints among needles in two dimensions respect to 3D. 


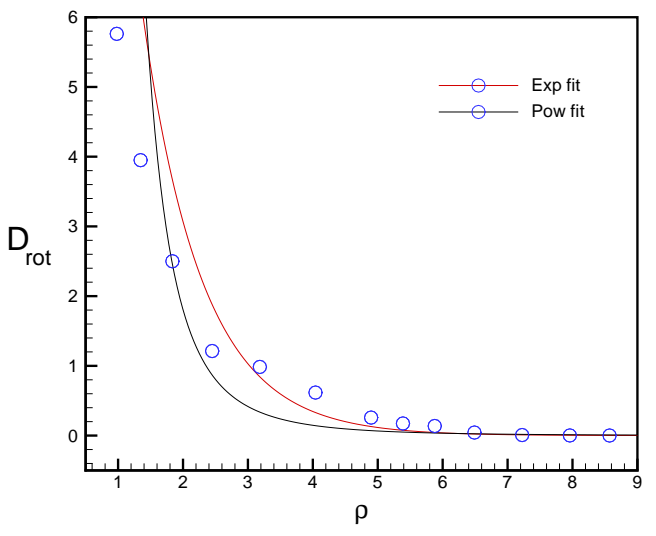

FIG. 11: Fig.13: Dependence of $D_{\text {rot }}$ on $\rho$.

\section{SUMMARY AND CONCLUDING REMARKS}

We have simulated the dynamics of a $2 \mathrm{D}$ gas of hard needles by event-oriented molecular dynamics. Many of the temporal autocorrelation functions both translational and angular exhibit a sort of slow dynamics and multi step relaxation. The most interesting feature is the exis- tence of three regimes in the angular mean squared displacement. This can be attributed to slow dynamics. Our findings show relaxing the translational degrees of freedom does not smear out angular slow dynamics. Density dependence of translational and rotational diffusion coefficients has been obtained and compared to three dimensional results. In 2D dependence of the translational diffusion coefficient on density qualitatively resembles to 3D. Rotational diffusion constant exhibits an algebraic decay but with a larger exponent than in $3 \mathrm{D}$.

\section{ACKNOWLEDGEMENT}

This work has been funded by Iran National Science Foundation under the grant number 844169 . We would like to deeply express our gratitude to Mainz university for the kind hospitality and computing facility during our visit to Professor Kurt Binder group where parts of this project were carried out. We are highly indebted to Tanja Schilling for very stimulating and fruitful discussions and enlightening comments. Enlightening comments of an anonymous referee is appreciated. Useful discussions with Mir Faez Miri is appreciated. MEF is thankful to B. Vazirol Vozaraa for useful help.
[1] M. P. Allen, D. Frenkel and J. Talbot, Computer Phys. Reports, 9, 301 (1989).

[2] P. G. de Gennes and J. Prost, The Physics of Liquid Crystals, 2nd Ed., Oxford University Press 1995.

[3] D. Frenkel, Mol. Phys., 60, 1 (1987).

[4] P. Pfleiderer, K. Milinkovic and T. Schilling, Euro. Phys. Lett., 84, 16003 (2008).

[5] M. Doi, I. Yamamoto and F. Kano, J. Phys. Soc. Jpn, 53, 3000 (1984).

[6] M. Fixman, Phys. Rev. Lett., 54, 337 (1985).

[7] Y. G. Tao, W. K. den Otter, J. K. G. Dehont and W. J. Briels, J. Chem. Phys, 124, 134906 (2006).

[8] M. Huthmann, T. Aspelmeier and A. Zippelius, Phys. Rev. E, 60, 654 (1999).

[9] M. J. Green, R. A. Brown and R. C. Armstrong, J. Chem. Phys, 126, 034903 (2007).

[10] J. J. Magda, H. T. Davis and M. Tirrell, J. Chem. Phys, 104, 6755 (1996).

[11] H. H. Wensink and R. L. C. Vink, J. Phys.: Condensed Matter, 19, 466109 (2007).

[12] A. Chrzanowska , Acta. Phys. Polonica B, 36, 3163 (2005).

[13] M. Otto, T. Aspelmeier and A. Zippelius, J. Chem. Phys, 124, 154907 (2006)

[14] D. Frenkel and J. F. Maguire, Phys. Rev. Lett., 47, 1025 (1981).

[15] D. Frenkel and J. F. Maguire, Mol. Phys, 49, 503 (1983).

[16] J. J. Magda, H. T. Davis and M. Tirrell, J. Chem. Phys, 85, 6674 (1986).
[17] D. Frenkel and R. Eppenga, Phys. Rev. A, 31, 1776 (1985).

[18] M. Kosterlitz and D. Thouless, J. Phys. C, 6, 1181 (1973).

[19] C. Renner, H. Löwen and J. L. Barret, Phys. Rev. E, 52, 5091 (1995).

[20] S. Obukhov, D. Kobzeb, D. Perchak and M. Rubinstein, J. Phys. I France, 7, 563 (1997).

[21] C. A. Angell, J. H. R. Clarke and L. V. Woodcock, Adv. Chem. Phys. 48, 397 (1981).

[22] A. Chrzanowska and H. Ehrentraut, Phys. Rev. E, 66, 012201 (2002).

[23] A. Chrzanowska, J. Chem. Phys, 120, 2857 (2004).

[24] A. Mukôyama and Y. Yoshimura, Molecular Simulation, 21, 367 (1998).

[25] A. Mukôyama and Y. Yoshimura, J. Phys. A: Math. Gen., 34, 4053 (2001).

[26] M. Jiménez-Ruiz, A. Criado, F. J. Bermejo, G. J. Cuello et al. J. Phys.: Condens. Matter, 14, 1509 (2002).

[27] M. D. Khandkar and M. Barma, Phys. Rev. E, 72, 05717 (2005)

[28] Y. Kantor and M. Kardar, Phys. Rev. E, 79, 041109 (2009).

[29] K. Raghavan, Molecular Physics, 84, 345 (1995).

[30] K. M. Sando and D. W. Rebertus, J. Chem. Phys, 67, 2585 (1977).

[31] J. Talbot, M. P. Allen, G. T. Evans, D. Frenkel and D. Kivelson, Phys. Rev. A, 39, 4330 (1989). 


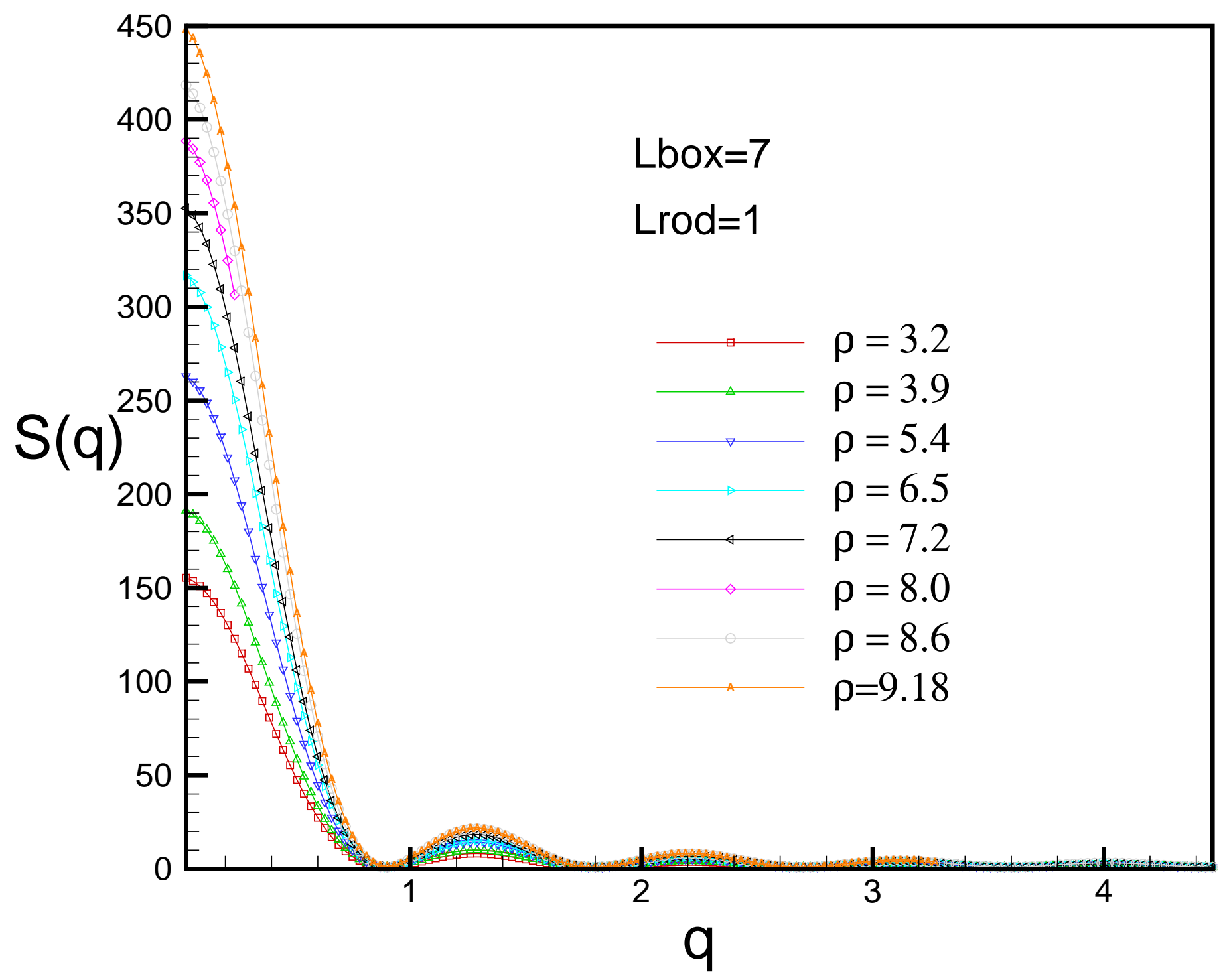

